\title{
Evaluating denture cleanliness of patients in a regional dental hospital
}

\author{
P. Mylonas, ${ }^{* 1}$ D. C. Attrill ${ }^{2}$ and A. D. Walmsley ${ }^{3}$
}

\section{In brief}

Outlines the use of the Denture Cleanliness Index in a secondary dental care setting.
The methodology and results are applicable for use by clinicians in a secondary care setting when evaluating patients' denture hygiene.
Shows how educational intervention can help our patients.

Aims To evaluate the quality of clinical record keeping and determine quality of denture cleanliness; record baseline denture cleanliness for 60 patients; introduce denture hygiene instruction (DHI); and then re-assess the patients for improvement and enhanced record keeping. Methods Analysis of patients' denture hygiene instruction record keeping $(n=60)$ was undertaken retrospectively. The Denture Cleanliness Index (DCI) was utilised to assess denture cleanliness (best score 0, worst score 4). Baseline DCI scores were taken and individual DHI was delivered. After one month, patients were reviewed and scored, with record keeping quality analysed. Results At baseline, $11.7 \%(n=7)$ of patients had DCl scores of $\leq 2$, improving to $93.8 \%$ $(n=45)$ after one month, demonstrating short-term improvement in denture cleanliness. Only $63 \%(n=38)$ of patients had evidence of a record of DHI within their notes at baseline, improving to $100 \%$ at recall. Conclusions New patient information leaflet and clinical guidelines on denture hygiene have been written and implemented. The results of this study suggest that this may be a relatively straightforward method to achieve a short-term improvement in denture cleanliness. The implementation of a DCI score is helpful in allowing patients to improve denture hygiene and its wider use is supported.

\section{Introduction}

In order to achieve optimal oral health it is well documented in the literature that denture wearing patients should be advised to maintain high standards of both oral hygiene and denture hygiene. ${ }^{1-6}$

Dentures can accumulate plaque and develop calculus deposits in a manner similar to natural teeth, and the composition of denture plaque differs when compared with dental plaque. Studies have shown that denture plaque can contain a number of potentially harmful microorganisms including: Methicillin-resistant Staphylococcus aureus, Candida albicans, and Steptococcus mutans..$^{7-11}$

'Dental Core Trainee in Restorative Dentistry. Birmingham Dental Hospital, St Chad's Queensway, Birmingham, B4 6NN; ${ }^{2}$ Senior Lecturer and Honorary Consultant in Restorative Dentistry, ${ }^{3}$ Professor and Honorary Consultant in Restorative Dentistry, School of Dentistry, College of Medical and Dental Sciences, University of Birmingham, St Chad's Queensway, Birmingham, B4 6NN

${ }^{*}$ Correspondence to: Mr Petros Mylonas

Email: p.mylonas@bham.ac.uk

Refereed Paper. Accepted 20 June 2016

DOI: $10.1038 /$ sj.bdj.2016.562

${ }^{\oplus}$ British Dental Journal 2016; 221: 127-130
Previous studies indicate that inadequate denture hygiene can lead to an increase in accumulation of denture plaque, which can then increase the risk of developing oral and dental diseases such as dental decay, periodontal disease, and denture stomatitis. ${ }^{4,10-13}$

Unfortunately many patients have been shown to have inadequate denture hygiene and some still wear their dentures at night, despite evidence that nocturnal denture wearing is linked to a decrease in oral health and provides no benefit to patients' quality of sleep. ${ }^{5,12,14,15}$

A number of different cleaning regimes are available for patients to clean their dentures and they can be classified into either chemical or mechanical methods. According to a number of different studies and surveys evaluating the denture hygiene habits of patients, the most commonly used mechanical method is a toothbrush with soap and water or toothpaste, while the most commonly used chemical method is either sodium hypochlorite-based cleaner or a peroxide-based cleaner. ${ }^{1,2,5,14,16,17}$

Evidence from studies conducted outside the UK provides evidence of the denture care habits and the quality of denture hygiene of non-UK cohorts. Their results may potentially be used to inform on the denture care advice given to UK denture wearers, however, there likely will be potential differences in the availability of denture care materials and in patient denture care/hygiene attitudes. Few studies and surveys have been conducted in the UK which outlines the quality of patients' denture hygiene, their denture cleaning habits, and the quality of clinical record keeping.

The purpose of this paper is to outline the results of a clinical audit conducted in the prosthodontics department of a regional dental hospital. It follows on from work conducted within general dental practice and seeks to determine whether denture hygiene differs between a primary care and secondary care cohort; this comparison has yet to be published in the available literature. ${ }^{2}$

\section{Methodology}

Sixty consecutive denture wearing patients were selected opportunistically, attending for treatment at the Prosthodontics Department of the Birmingham Dental Hospital.

Acrylic denture wearers only, both complete and partial, were included in the 
Fig. 1 Quality of record keeping at baseline

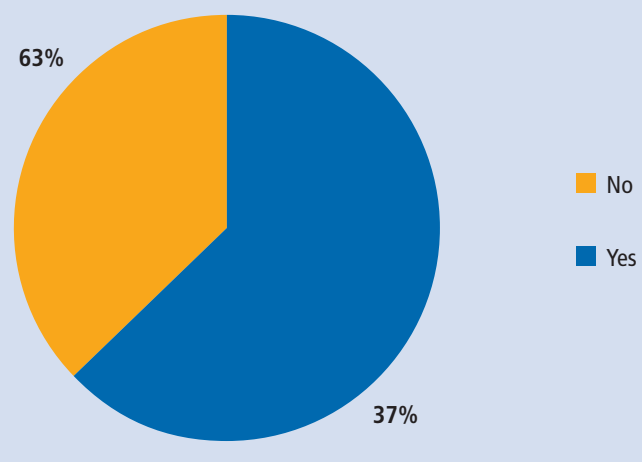

\section{Table 1 The Denture Cleanliness Index (Mylonas et al., 2014)}

\begin{tabular}{l|l} 
Index & Clean denture. No plaque is visibly seen, no staining, no plaque detectable \\
\hline 0 & $\begin{array}{l}\text { Denture is visibly clean. Little staining } \\
(<25 \% \text { staining of fit surface) }\end{array}$ \\
\hline 2 & $\begin{array}{l}\text { Denture has visible plaque and/or debris. Moderate staining of fit surface } \\
(25-50 \% \text { staining of the fit surface) }\end{array}$ \\
\hline 3 & $\begin{array}{l}\text { Denture has visible plaque and/or debris. Severe staining of fit surface } \\
(>50 \% \text { staining of the fit surface) }\end{array}$ \\
\hline 4 & $\begin{array}{l}\text { Denture has visible calculus deposit(s), on any surface } \\
\text { Visible defects in denture, in addition to any of the above score. } \\
\text { (Defects defined as those which are potentially plaque retentive, those which require repair } \\
\text { or remake of denture) }\end{array}$ \\
\hline$*$ &
\end{tabular}

audit to maintain the simplicity of the audit. All patients were examined by one clinician. Informed consent was obtained from each patient before assessment.

\section{Audit standard}

Acceptable denture hygiene was defined as Denture Cleanliness Index (DCI) score of 2 or less. For patients observed, $90 \%$ or greater should have acceptable denture hygiene. One hundred percent of clinical notes should have evidence of denture hygiene instructions (DHI) recorded.

\section{Patient examination}

Quality of patient denture hygiene was assessed using the DCI (Mylonas et al. 2014), which grades the severity of denture hygiene according to the amount of staining on the denture fitting surface. A liquid plaque disclosing dye (Plaqsearch, Malmö, Sweden) was applied by the clinician to the fitting surface as this is where denture plaque is most likely to accumulate ${ }^{18}$ and therefore the aspect of denture where patients need to clean most effectively. The stained denture was then assessed according to the DCI rubric (Table 1), with scores ranging from 0 (best) to 4 (worst).

\section{Patient assessment}

Patients' baseline DCI scores were obtained, their clinical records were evaluated, and tailored denture hygiene instructions were provided. They were then reviewed after one month, and their denture cleanliness was reassessed to obtain second audit cycle results for their DCI scores; patients' clinical records were also reassessed for quality of record keeping.

A patient information leaflet was written according to principles from Weinman (1990) and Mylonas et al. (2014), and given to patients to aid in patient education. ${ }^{2,19}$ The instructional leaflet detailed the importance of cleaning dentures and teeth, the frequency of cleaning, the manual and chemical methods according to the type of material it is made from and whether it has been relined.

For patients requiring a denture care pack (GlaxoSmithKlein, Brentford, Middlesex) these were provided and they contained a denture box and brush, a sample of effervescent tablets, a sample of denture adhesive cream and associated instructional leaflet.

\section{Results}

Sixty patients were seen for baseline assessment of their denture hygiene and their clinical records were evaluated for evidence that denture hygiene instructions being given; $26(43.3 \%)$ were male and $34(56.7 \%)$ were female, ranging in age between 18 to 84 with a mean age of 63.1 years. At one month review 12 patients did not turn up for their review appointment, despite multiple attempts to contact patients and remind them of their review appointments, and 48 patients were seen for review.

\section{Record keeping}

Results for the first cycle/baseline can be seen in Figure 1 and show that $63.3 \%(n=38)$ of patient notes had evidence of denture hygiene instructions being given recorded, while 36.7\% did not have any evidence at all. From the $63.3 \%$ of patient notes where DHI had been given, there was no evidence of standardisation of denture care instructions provided to patients.

After one month review, $100 \%$ of clinical notes had evidence of DHI being given after written instructions to be included in clinical notes was standardised.

\section{Denture hygiene and cleanliness}

At baseline $88.3 \%(n=53)$ patients had DCI scores of 3 or greater: $50 \%(n=30)$ with DCI score 3 and $38.3 \%(n=23)$, which is poor when compared to the audit standard set (Fig. 2).

After educational intervention, providing patients with tailored denture hygiene instruction, one month review results can be seen in Figure 3. It can be seen that patients' DCI scores improved, with $93.8 \%(n=45)$ having DCI scores 2 or less which equates to $75 \%$ $(\mathrm{n}=36)$ with DCI 2 and $18.8 \%(\mathrm{n}=9)$ DCI 1 , as can be seen in Figure 3 .

\section{Discussion}

Patients with removable dentures must be able to look after their prostheses properly and demonstrate good denture hygiene, as well as good oral hygiene, in order to maintain optimal oral health and minimise the risks of developing oral diseases. ${ }^{1-6}$ 
Fig. 2 Quality of denture cleanliness at baseline

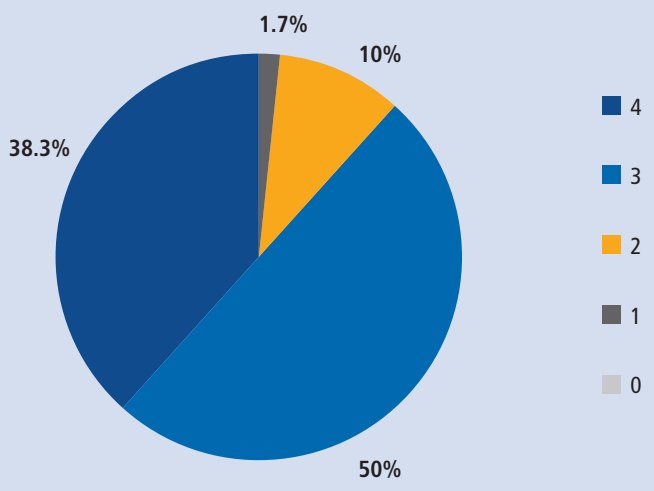

Fig. 3 Quality of denture cleanliness at one month review

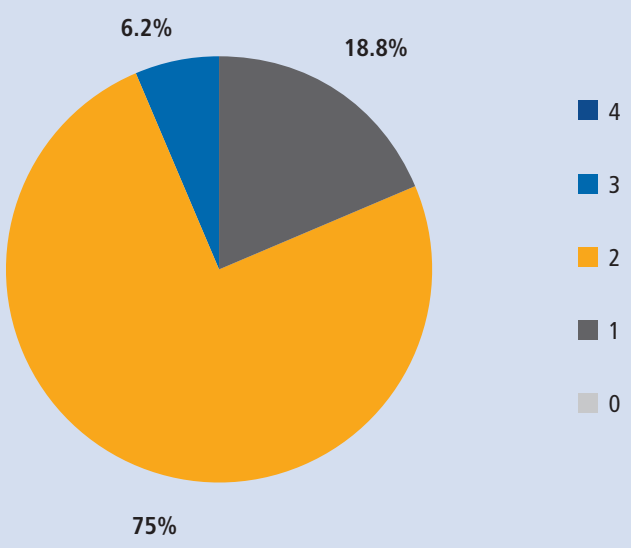

The results of this clinical audit show that the quality of patients' denture hygiene at baseline was unfortunately inadequate, which was consistent with another audit conducted on patients within the primary dental care setting and with other studies. ${ }^{2,5,14,20}$ While the baseline results for patients in this audit was slightly better than for those seen in primary dental care, the reasons for the inadequacy of patient denture hygiene were similar: lack of standardisation of denture care instructions, and lack of evidence that denture hygiene instructions were previously given to patients.

Patients were provided with tailored educational intervention in the form of denture hygiene instructions and leaflets, and a denture care pack for those that required it. After intervention, one month results indicated that patients' denture hygiene improved and exceeded the audit standard. The effects of educational intervention also mirror those improvements seen within the primary care audit by Mylonas et al. and highlights the positive effects of patient education on the subsequent quality of the denture hygiene. ${ }^{2,5,14}$ These results differ from those of Burnett et al. in 1993, who found that after six months of educational intervention - written and verbal - there was no change in the denture cleaning habits of their subject group. Conversely, it has been demonstrated that illustrated denture instruction manuals and frequent recall intervals - with denture hygiene and oral hygiene reinforcement - aids in improving denture and oral hygiene habits, and can therefore be recommended. ${ }^{21-23}$

The quality of clinical record keeping, at baseline, did not meet the clinical audit standards and after standardisation of terminology, improved dramatically at one month review, in accordance with similar intervention in another audit conducted in primary care. ${ }^{2}$

Patients were advised to use both chemical and mechanical cleaning methods to optimise their denture hygiene, in agreement with current literature, following similar advice given by other authors. ${ }^{24-26}$

The Denture Cleanliness Index provided a simple and quick method for evaluating the quality of denture hygiene within the secondary care setting, and allowed for standardisation of clinical record keeping with regards to denture hygiene assessment and denture hygiene instructions provided to patients. These results are similar to other studies where authors utilised their own methods for evaluating denture plaque. ${ }^{5,11,27}$ Patients could potentially be provided with disclosing solution for home-use in order to facilitate denture biofilm, however a study conducted in Brazil concluded that the provision of disclosing solutions for home-use by denture-wearing patients did not improve their ability to remove biofilm. ${ }^{28}$ However, there is evidence to support that providing patients with disclosing agents for home-use improves their oral plaque control irrespective of whether they are undergoing general dental review, ${ }^{29}$ active periodontal treatment, ${ }^{30}$ or orthodontic fixed-appliance treatment. ${ }^{31}$ Further research will be needed to ascertain the effectiveness of denture care instructions with concomitant home-use of disclosing agents in improving denture wearing patients' ability to remove denture biofilm.

As clinicians we are obligated to provide patients with the necessary information and motivation required to look after their dentures as well as assess patients' compliance to said instructions as by doing so this can improve patients' oral and denture plaque control. ${ }^{21,22}$

\section{Conclusions}

Denture wearers treated within a secondary care environment exhibited slightly better levels of denture hygiene compared with those in primary care, but the levels of denture hygiene were deemed to be inadequate overall.

Patient education on appropriate denture hygiene care led to an improvement in their overall denture cleanliness, and must be reinforced at clinical examination.

The Denture Cleanliness Index provides the clinician with an easy tool to assess denture cleanliness, provide tailored denture hygiene instruction, and assess patient compliance.

Denture hygiene instructions and oral hygiene instructions should be provided to all denture wearing patients to reduce the chances of developing oral disease.

1. Jagger $D$, Harrison A. Denture cleansing - the best approach. Br Dent J 1995; 178: 413-417.

2. Mylonas P, Afzal Z, Attrill D. A clinical audit of denture cleanliness in general dental practice undertaken in the West Midlands. Br Dent J 2014; 217: 231-234.

3. Saha A, Dutta S, Varghese R K, Kharsan V, Agrawal A. A survey assessing modes of maintaining denture hygiene among elderly patients. J Int Soc Prev Community Dent 2014; 4: 145-148. 
4. Kulak-Ozkan Y, Kazazoglu E, Arikan A. Oral hygiene habits, denture cleanliness, presence of yeasts and stomatitis in elderly people. J Oral Rehabil 2002; 29: 300-304.

5. Dikbas I, Koksal T, Calikkocaoglu S. Investigation of the cleanliness of dentures in a university hospital. Int Prosthodont 2006; 19: 294-298.

6. de Castellucci Barbosa L, Ferreira M R M, de Carvalho Calabrich C F, Viana AC, de Lemos M C L, Lauria R A. Edentulous patients' knowledge of dental hygiene and care of prostheses. Gerodontology 2008; 25: 99-106.

7. Preshaw P M, Walls a. WG, Jakubovics N S, Moynihan P J, Jepson N J, Loewy Z. Association of removable partial denture use with oral and systemic health. J Dent 2011; 39: 711-719.

8. Sumi Y, Kagami H, Ohtsuka Y, Kakinoki Y, Haruguchi $Y$, Miyamoto H. High correlation between the bacterial species in denture plaque and pharyngeal microflora. Gerodontology 2003; 20: 84-87.

9. Sumi Y, Miura H, Michiwaki Y, Nagaosa S, Nagaya M. Colonization of dental plaque by respiratory pathogens in dependent elderly. Arch Gerontol Geriatr 2007; 44: 119-124.

10. Coulthwaite L, Verran J. Potential pathogenic aspects of denture plaque. Br J Biomed Sci 2007; 64: 180-189.

11. Budtz-Jørgensen E, Bertram V. Denture stomatitis I: The aetiology in relation to trauma and infection. Acta Odontol Scand 1970; 28: 71-92.

12. Emami E, Taraf $\mathrm{H}$, de Grandmont $\mathrm{P}$ et al. The association of denture stomatitis and partial removable dental prostheses: a systematic review. Int J Prosthodont 2012; 25: 113-119.

13. Fiske J, Davis D, Horrocks P. A self-help group for complete denture wearers. Br Dent J 1995; 178: 18-22.
14. Ferruzzi F, Martins J C L S, Corrêa G O, Kurihara E, Sábio $S$. Effects of hygiene guidance associated or not to provision of hygiene devices on habits of denture wearers. Acta Sci Heal Sci 2015; 37: 77.

15. Takamiya A, Monteiro D, Barão V, Pero A, Compagnoni $M$, Barbosa D. Complete denture hygiene and nocturnal wearing habits among patients attending the Prosthodontic Department in a Dental University in Brazil. Gerodontology 2011; 28: 91-96.

16. Uludamar A, Ozkan Y K, Kadir T, Ceyhan I. In vivo efficacy of alkaline peroxide tablets and mouthwashes on Candida albicans in patients with denture stomatitis. J Appl Oral Sci 2010; 18: 291-296.

17. Cakan U, Yuzbasioglu E, Kurt $\mathrm{H}$ et al. Assessment of hygiene habits and attitudes among removable partial denture wearers in a university hospital. Niger J Clin Pract 2015; 18: 511-515.

18. Keng S, Lim M. Denture plaque distribution and the effectiveness of a perborate-containing denture cleanse. Quintessence Int (Berl) 1996; 27: 341-345.

19. Weinman J. Providing written information for patients: psychological considerations. J R Soc Med 1990; 83: 303-305.

20. Ogunrinde T, Opeodu O. Denture care practice among patients attending the prosthetic clinic in a Nigerian teaching hospital. Niger Med J 2015; 56: 199-203.

21. Ribeiro D, Pavarina A, Giampaolo E, Machado A, Jorge J, Garcia P. Effect of oral hygiene education and motivation on removable partial denture wearers: longitudinal study. Gerodontology 2009; 26: 150-156.

22. Koyama S, Hanabuchi S, Fuji T et al. The difference between baseline and 5 -year examinations at recall in $P C R$, PD, tooth mobility, and BRL of abutment teeth in subjects who had received periodic maintenance care more than four times per year. Annals Japan Prosthodon Soc 2012; 4: 59-67.

23. Burnett C A, Calwell E, Clifford T J. Effect of verbal and written education on denture wearing and cleansing habits. Eur J Prosthodont Restor Dent 1993; 2: 79-83.

24. Cruz P C, Andrade I M De, Peracini A et al. The effectiveness of chemical denture cleansers and ultrasonic device in biofilm removal from complete dentures. J App/ Oral Sci 2011; 19: 668-673.

25. Fernandes R, Lovato-Silva C, Paranhos H, Ito I. Efficacy of three denture brushes on biofilm removal from complete dentures. J Appl Oral Sci 2000; 15: 39-43.

26. Salles A, Macedo L, Fernandes R, Silva-Lovato C, Paranhos $\mathrm{H}$. Comparative analysis of biofilm levels in complete upper and lower dentures after brushing associated with denture paste and neutral soap. Gerodontology 2007; 24: 217-223.

27. Taiwo J O, Arigbede A O, Harcourt P. Denture hygiene of the elderly denture wearers in South East local government area in Ibadan, Nigeria. J Biol Agric Healthc 2012; 2: 22-27.

28. Souza R, Regis $R$, Nascimento C, Paranhos $H$, Lorato Silva $\mathrm{H}$. Domestic use of a disclosing solution for denture hygiene: a randomised trial. Gerodontology 2010; 27: 193-198.

29. Nepale M, Varma S, Suragimath G, Abbayya K, Zope S, Kale $V$. A prospective case-control study to assess and compare the role of disclosing agent in improving the patient compliance in plaque control. J Oral Res Rev 2014; 6: 45-48.

30. Frazao P, Sammarone M, Villa S. Effect of disclosing agents in oral hygiene. Cienc Odontol 2004; 1: 52-59.

31. Peng Y, Wu R, Qu W et al. Effect of visual method vs plaque disclosure in enhancing oral hygiene in adolescents and young adults: a single-blind randomized controlled trial. Am J Orthod Dentofacial Orthop 2014; 145: $280-286$. 